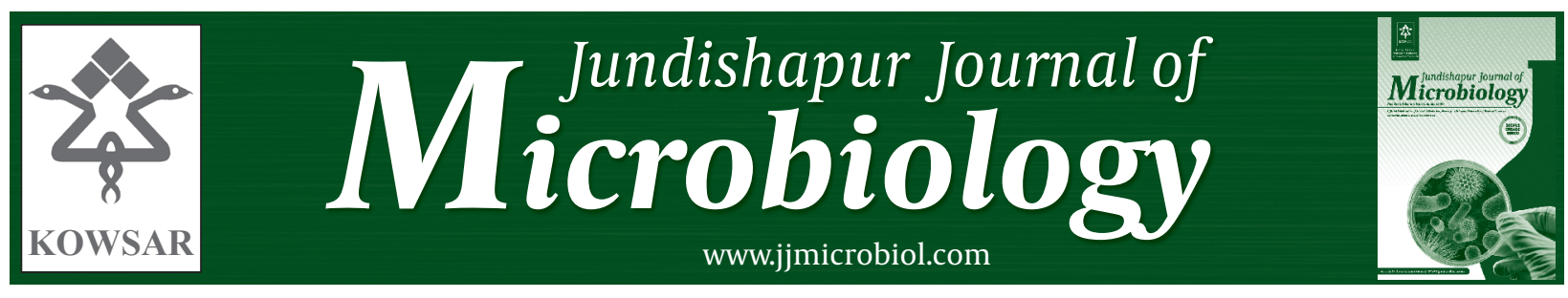

\title{
Sensitivity of Vaginal Isolates of Candida to Eight Antifungal Drugs Isolated From Ahvaz, Iran
}

\author{
Zahra Salehei ${ }^{1}$, Zahra Seifi ${ }^{1^{*}}$, Ali Zarei Mahmoudabadi ${ }^{1,2}$ \\ ${ }^{1}$ Department of Medical Mycology, School of Medicine, Ahvaz Jundishapur University of Medical Sciences, Ahvaz, IR Iran \\ 2 Infectious Diseases and Tropical Medicine Centre, Ahvaz Jundishapur University of Medical Sciences, Ahvaz, IR Iran
}

\begin{tabular}{l}
\hline A R T I C L E I N F O \\
\hline Article type: \\
Original Article \\
\hline Article history: \\
Received: 21 Feb 2012 \\
Revised:10 Mar 2012 \\
Accepted: 03 Apr 2012 \\
\hline
\end{tabular}

Keywords:

Candida albicans

Candidiasis Vulvovaginal

Antifungal Agents

Microbial Sensitivity Tests

\begin{abstract}
A B S T R A C T
Background: Candida vaginitis is a common fungal infection among adult women and it has been estimated that $75 \%$ of all adult women experience at least one period of vulvovaginal candidiasis in their lifetime. Several predisposing factors, such as diabetes mellitus, using contraceptive, pregnancy, and broad-spectrum antibiotics are reported as main risk factors for the infection. While, the main etiologic agent of vulvovaginal candidiasis is Candida albicans, more antifungal resistance has been reported among non-albicans species.

Objectives: The aim of the present study was to determine susceptibility patterns of vaginal isolates of Candida to eight antifungal drugs including, clotrimazole, miconazole, terbinafine, nystatin, itraconazole, fluconazole, ketoconazole, and econazole.

Patients and Methods: Tested organisms were C. albicans 53 (79.1\%), C. glabrata 8 (11.9\%), C. tropicalis $4(5.9 \%)$ and C. krusei $2(2.9 \%)$ that were isolated from vaginal infected patients. Disk diffusion method was used to evaluate susceptibility patterns. Paper disks containing clotrimazole, miconazole, itraconazole, fluconazole, ketoconazole, econazole, nystatin and terbinafine were applied for susceptibility tests.

Results: In the present study 2 isolates of $C$. krusei were sensitive to ketoconazole, clotrimazole and miconazole. In addition both isolates were resistant to fluconazole, nystatin, econazole and terfinafine. Only one isolate of C. tropicalis was sensitive to miconazole and terbinafine and two isolates to clotrimazole. Highest sensitivity of $C$. albicans to antifungal drugs was seen against miconazole (49 of 53 isolates) followed by, clotrimazole (41), terbinafine (28) and ketoconazole (13) whereas 43 isolates were resistant to fluconazole and econazole antifungals.

Conclusions: All 8 isolates of C. glabrata were resistant to fluconazole, whereas all isolates were sensitive to miconazole. Antifungal sensitivity testing suggests that vaginal isolates of Candida were most sensitive to miconazole, clotrimazole, and terbinafine, and least sensitive to econazole and fluconazole.
\end{abstract}

Published by Kowsar Corp, 2012. cc 3.0.

Implication for health policy/practice/research/medical education:

Fluconazole resistance was the most prevalent (85.1\%) among all non-Candida albicans species and also the majority isolates of $C$. albicans. In addition terbinafine is presented as one of the choices for the treatment of Candida vaginitis.

Please cite this paper as:

Salehei Z, Seifi Z, Zarei Mahmoudabadi A.Sensitivity of Vaginal Isolates of Candida to Eight Antifungal Drugs Isolated From Ahvaz, Iran. Jundishapur J Microbiol. 2012;5(4):574-7. DOI:10.5812/jjm.4556.

* Corresponding author: Zahra Seifi, Department of Medical Mycology, School of Medicine, Ahvaz Jundishapur University of Medical Sciences, Ahvaz, IR Iran, Tel: +98-6113330074, Fax:+98-6113332036,E-mail: zseifi@hotmail.com

DOI: $10.5812 / j j m .4556$

(C) 2012 Ahvaz Jundishapur University of Medical Sciences; Published by Kowsar Corp.

This is an Open Access article distributed under the terms of the Creative Commons Attribution License (http://creativecommons.org/licenses/by/3.0), which permits unrestricted use, distribution, and reproduction in any medium, provided the original work is properly cited. 


\section{Background}

Vulvovaginal candidiasis (VVC) or Candida vaginitis is a common fungal infection among adult women during reproductive ages. It has been estimated that $75 \%$ of all adult women experience at least one period of vulvovaginal candidiasis in their lifetime (1). fortunately the infection is rarely life threatening, whereas it is usually associated with such morbidities like discomfort, pain, sexual dysfunctions, vulvar dryness, cracks, itching, burning, soreness and finally health care costs (2-4). Known predisposing host factors, which include uncontrolled diabetes mellitus, using contraceptive, compromised immune system, neutropenia, pregnancy, hormone replacement therapy and broad-spectrum antibiotics are risk factors for VVC $(1,5)$.

Several reports have shown that prevalence of vulvovaginal candidiasis in Iran is remarkable and similar to other parts of the world (6-10). However, there are a few reports about susceptibility of vaginal isolates to antifungal agents in vitro circumstances. In addition some studies have shown that there are different results from treatment of vulvovaginal candidiasis (9, 11-15). There are also several reports indicating that resistance to antifungals, and infection recurrent is a serious problem among Iranian patients $(11,12,14)$. In a study performed in Qazvin, authors believed that there is no significant difference between fluconazole and clotrimazole resistance in recurrent candidiasis (9). Prolonged therapy and increased use of antifungals for recurrent candidiasis are the most common risk factors for azoles resistance among Candida isolates from vulvovaginitis candidiasis patients. Azoles have the advantage of being taken orally, which increase their potency $(2,4)$.

The inappropriate use of antifungal drugs and introduction of over-the-counter antimycotics in countries worldwide predispose development of antifungal resistance (6). In a study conducted by Richter et al., fluconazole resistance was observed among $15.2 \%$ and $41.7 \%$ of vaginal isolates of C. glabrata and C. krusei, respectively (1). Whereas resistance to itraconazole was observed in non-albicans species, C. glabrata, C. parapsilosis, C. krusei, and $S$. cerevisiae isolates (1). In another study, vaginal isolates of Candida were more dose-dependent susceptible to nystatin and ketoconazole (16). Candida species are the normal microbiota within the oral cavity, gastrointestinal tracts, respiratory tracts, vaginal area and the mouth (4). The majority of cases of vulvovaginal candidiasis are caused by C. albicans, other etiologic agents are C. glabrata, C. tropicalis and C. krusei $(1,4,17)$. However, Mohanty et al. has reported C. glabrata as the main etiology of vulvovaginal candidiasis (13).

\section{Objectives}

This study was carried out to determine susceptibility patterns of vaginal isolates of Candida species to eight antifungals including, clotrimazole, miconazole, itraconazole, fluconazole, ketoconazole, econazole, terbinafine, and nystatin.

\section{Patients and Methods}

\subsection{Isolates and Identification}

In the present study, 67 vulvovaginal isolates of Candida species were investigated for performing susceptibility tests. The isolates were kept in sterile distilled water at $4{ }^{\circ} \mathrm{C}$ in the medical mycology laboratory of Ahvaz Jundishapur University of medical sciences. The most common isolate was C. albicans $(53,79.1 \%)$ followed by C. glabrata (8, 11.9\%), C. tropicalis $(4,5.9 \%)$ and C. krusei $(2,2.9 \%)$. All isolates were re-identified using standard methods, CHROMagar Candida (CHROMagar Candida $®$, Paris, France) germ tube test and microscopic characteristics on corn meal agar (HiMedia, India). Identification was based on colonies producing a green coloration which were presumptively identified as C. albicans (17). Germ tube test, production of chlamydoconidia and growing at $45^{\circ} \mathrm{C}$ were also a confirmation for the isolates (17). C. glabrata produced pink colonies on CHROMagar Candida which microscopic characteristics on corn meal agar confirmed it (17). Dark blue coloration on CHROMagar Candida and microscopic features confirmed C. tropicalis. Pale pink and spread colonies on CHROMagar Candida and microscopic features on Cornmeal agar were identical for C. krusei.

\subsection{Antifungal Disks}

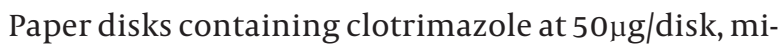
conazole at $10 \mu \mathrm{g} / \mathrm{disk}$, itraconazole $50 \mu \mathrm{g} / \mathrm{disk}$, fluconazole $100 \mu \mathrm{g} / \mathrm{disk}$, ketoconazole $10 \mu \mathrm{g} / \mathrm{disk}$, econazole $10 \mu \mathrm{g} /$ disk, and nystatin 100U/disk were obtained from Liofilchem Bacteriology Products (Italy). Terbinafine

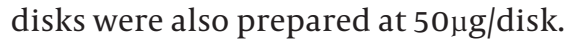

\subsection{Test Method}

A total of 67 Candida species were sub-cultured on Sabouraud's dextrose agar plates, SDA (Merck, Germany) and incubated at $37^{\circ} \mathrm{C}$ for $24 \mathrm{~h}$. A suspension of overnight cultures of C. albicans (53), C. glabrata (8) and C. tropicalis (4) and C. krusei (2) were prepared in sterile PBS. Turbidity was adjusted to 0.5 McFarland standard density resulting in an inoculum containing $1-5 \times 106$ $\mathrm{CFU} / \mathrm{ml}$. $25 \mu \mathrm{l}$ of suspension inoculated on SDA plates and rolled on the surface of the agar medium. Plates were dried for $15 \mathrm{~min}$ at room temperature in laminar hood and then antifungal disks were placed on the inoculated agar with a forceps. The plates were incubated at $37^{\circ} \mathrm{C}$ for $24 \mathrm{~h}$, and then zone diameters were measured manually. 


\begin{tabular}{lcll|}
\hline \multirow{2}{*}{ Table 1. Criteria of Susceptibility and Resistance of Antifungal Disks } \\
\cline { 2 - 4 } & \multicolumn{3}{c}{ Zone Diameter, mm } \\
\hline Nystatin & Sensitive & Dose Dependent & Resistance \\
\hline Fluconazole & $\geq 19$ & $17-24$ & $\geq 16$ \\
Ketoconazole & $\geq 30$ & $23-18$ & $\geq 14$ \\
Clotrimazole & $\geq 20$ & $12-19$ & $\leq 22$ \\
Miconazole & $\geq 20$ & $12-19$ & $\leq 11$ \\
Itraconazole & $>16$ & $10-15$ & $<11$ \\
Econazole & $\geq 30$ & $23-29$ & $\leq 22$ \\
Terbinafine & $\geq 20$ & $12-19$ & $\leq 11$ \\
\hline
\end{tabular}

\section{Results}

In the present study susceptibility testing was performed on vaginal isolates of Candida collected during 2008 to 2009 from patients suspected to vulvovaginal candidiasis in Ahvaz, the capital city of Khuzestan. Criteria for susceptibility to used antifungal drugs have been summarized in Table 1(17-19). In the present study, several topical and systemic antifungal drugs were evaluated against 53 isolates of C. albicans, 8 C. glabrata, 4 C. tropicalis and 2 C. krusei. In our study two isolates of C. krusei were sensitive to ketoconazole, clotrimazole and miconazole. In addition both isolates were resistant to fluconazole, nystatin, econazole and terbinafine. Dose dependent was only observed about itraconazole. Only one isolate of $C$. tropicalis was sensitive to miconazole and terbinafine and two isolates were sensitive to clotrimazole (Table 2). jority of isolates (85.1\%) were resistant to fluconazole followed by $76.1 \%$ resistance to econazole (Figure). On the other hand, the best choice for the treatment of isolates was miconazole followed by clotrimazole, terbinafine and ketoconazole.

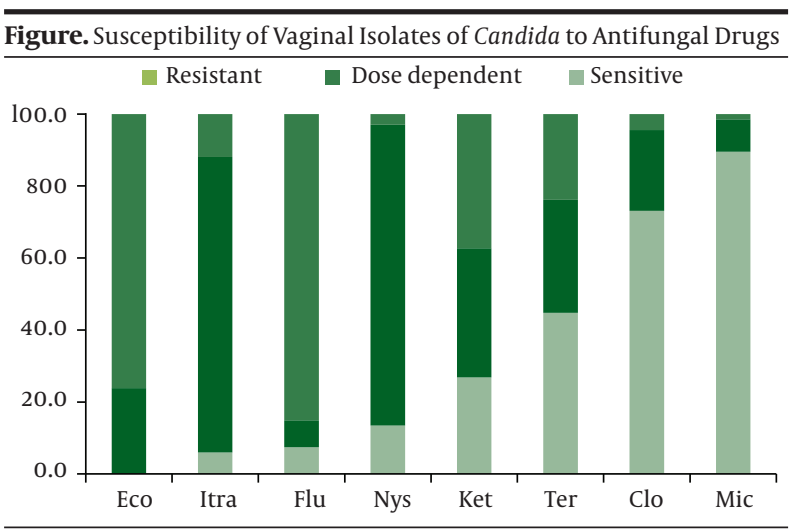

Abbreviations: Eco, Econazole; Itra, Itraconazole; Flu, Fluconazole; Nys, Nystatin; Ket, Ketoconazole; Ter, Terbinafine; Mic, Miconazole

\section{Discussion}

Vulvovaginal candidiasis is one of the most common opportunistic fungal infections among adult women during their lifetime. Several researches have shown that the infection increases during two to three last decades. Clotrimazole and fluconazole are the two antifungal drugs that are widely used in the treatment of vulvovaginal candidiasis. The main agent of vulvovaginal candidiasis is C. albicans; however, it seems non-albicans species

\begin{tabular}{|c|c|c|c|c|c|c|c|c|c|c|c|c|c|c|c|}
\hline & \multicolumn{3}{|c|}{ C. albicans } & \multicolumn{3}{|c|}{ C. glabrata } & \multicolumn{3}{|c|}{ C. tropicalis } & \multicolumn{3}{|c|}{ C. krusei } & \multicolumn{3}{|c|}{ Total, No. (\%) } \\
\hline & $S^{a}$ & DD $^{a}$ & $\mathbf{R}^{\mathbf{a}}$ & $S^{a}$ & DD $^{a}$ & $\mathbf{R}^{\mathbf{a}}$ & $S^{a}$ & DD $^{a}$ & $\mathbf{R}^{\mathbf{a}}$ & $S^{a}$ & DD $^{a}$ & $\mathbf{R}^{\mathbf{a}}$ & $S^{a}$ & DD $^{\mathrm{a}}$ & $\mathbf{R}^{\mathbf{a}}$ \\
\hline Itraconazole & 4 & 41 & 8 & 0 & 8 & 0 & 0 & 4 & 0 & 0 & 2 & 0 & $4(6.0)$ & $55(82.1)$ & $8(11.9)$ \\
\hline Fluconazole & 5 & 5 & 43 & 0 & 0 & 8 & 0 & 0 & 4 & 0 & 0 & 2 & $5(7.5)$ & $5(7.5)$ & $57(85.1)$ \\
\hline Ketoconazole & 13 & 17 & 23 & 3 & 4 & 1 & 0 & 3 & 1 & 2 & 0 & 0 & $18(26.9)$ & $24(35.8)$ & $25(37.3)$ \\
\hline Terbinafine & 28 & 18 & 7 & 1 & 3 & 4 & 1 & 0 & 3 & 0 & 0 & 2 & $30(44.8)$ & $21(31.3)$ & $16(23.9)$ \\
\hline Clotrimazole & 41 & 11 & 1 & 4 & 4 & 0 & 2 & 0 & 2 & 2 & 0 & 0 & $49(73.1)$ & $15(22.4)$ & $3(4.5)$ \\
\hline Miconazole & 49 & 4 & 0 & 8 & 0 & 0 & 2 & 2 & 0 & 2 & 0 & 0 & 60 (89.6) & $6(9.0)$ & $1(1.5)$ \\
\hline Nystatin & 7 & 46 & 0 & 2 & 6 & 0 & 0 & 4 & 0 & 0 & 0 & 2 & $9(13.4)$ & $56(83.6)$ & $2(3.0)$ \\
\hline Econazole & 0 & 10 & 43 & 0 & 4 & 4 & 0 & 2 & 2 & 0 & 0 & 2 & $0(0.0)$ & $16(23.9)$ & $51(76.1)$ \\
\hline
\end{tabular}

a Abbreviation: S, Sensitive; DD, Dose dependent; R, Resistant

All eight isolates of C. glabrata were resistant to fluconazole, whereas all isolates were highly sensitive to miconazole. Highest sensitivity of C. albicans to antifungal drugs was seen against miconazole (49 of 53 isolates) followed by, clotrimazole ( 41 of 53 ), terbinafine (28 of 53) and ketoconazole (13 of 53). 43 of 53 isolates were also resistant to fluconazole and econazole antifungals (Table 2). In the present study, ma-
(C. krusei and C. glabrata) of Candida appear to be increasing (20). C. glabrata is the second commonest agent in vaginal infections in most regions (20). The sensitivity patterns of Candida isolates varies among studies in different countries $(21,22)$ unfortunately it is shown that resistance to antifungal azoles has been increased in recent years (21). In addition, sources of Candida were also affecting on sensitivity to antifungal drugs $(17,21)$. 
Pelletier et al., believed that clotrimazole resistance was present in HIV-infected isolated of C. albicans (23). In Jordan, for example, Al-Abeid et al. showed that all tested Candida were susceptible to nystatin, miconazole, ketoconazole and fluconazole and C. albicans isolates were more susceptible to azoles than was $C$. glabrata (21). In addition, only $6 \%$ of $C$. albicans isolates were resistant to fluconazole. Quindos et al. study showed that $90.2 \%$ and $91.4 \%$ of isolates of Candida species were sensitive to fluconazole and ketoconazole, respectively (22). Whereas, our results show that $85.1 \%$ and $76.1 \%$ of tested isolates were resistant to fluconazole and econazole, correspondingly (Figure). Also our study shows that $100 \%$ of non-albicans species, were resistant to fluconazole, $64.3 \%$ to terbinafine and 57.1\% to econazole. Most of non-albicans Candida species have higher azoles MICs than albicans species (1). Terbinafine is a synthetic antifungal drug with fungicidal activity against dermatophytes, moulds and fungistatic activity against Candida species (24). Several researches have shown that terbinafine is the first choice for the treatment of dermatophytosis $(25,26)$, however, few details are available about its effects on vaginal isolates of Candida. Despite the fact our results show that terbinafine is effective against $76.1 \%$ of vaginal isolates. Therefore, terbinafine therapy can be considered as a good therapeutic option in the management of vaginal candidiasis. In conclusion antifungal sensitivity testing reveals that vaginal isolates of Candida were most sensitive to miconazole, clotrimazole, and terbinafine, and least sensitive to econazole, followed by fluconazole and ketoconazole.

\section{Acknowledgments}

We wish to thank the Department of Medical Mycology, Ahvaz Jundishapur University of Medical Sciences for supplying the clinical isolates of Candida species.

\section{Financial Disclosure}

None declared.

\section{Funding/Support}

None declared.

\section{References}

1. Richter SS, Galask RP, Messer SA, Hollis RJ, Diekema DJ, Pfaller MA. Antifungal susceptibilities of Candida species causing vulvovaginitis and epidemiology of recurrent cases. J Clin Microbiol. 2005;43 (5):2155-62.

2. Ehrstrom S, Yu A, Rylander E. Glucose in vaginal secretions before and after oral glucose tolerance testing in women with and without recurrent vulvovaginal candidiasis. Obstet Gynecol. 2006;108 (6):1432-7.

3. Nyirjesy P, Peyton C, Weitz MV, Mathew L, Culhane JF. Causes of chronic vaginitis: analysis of a prospective database of affected women. Obstet Gynecol. 2006;108 (5):1185-91.

4. Paulitsch A, Weger W, Ginter-Hanselmayer G, Marth E, Buzina W. A 5-year (2000-2004) epidemiological survey of Candida and non-Candida yeast species causing vulvovaginal candidiasis in Graz, Austria. Mycoses. 2006;49 (6):471-5.

5. Jilek P, Spacek J, Buchta V, Kucera Z, Drahosova M, Forstl M, et al. [Systemic immunity in patients with recurrent vulvovaginal candidiasis]. Ceska Gynekol. 2005;70 (6):453-9.

6. Aali BS, Tohidi A. Prevalence of Candida vaginitis among symptomatic patients in Kerman.J Qazvin Uni Med Sci. 2000;13:42-8.

7. Aghamirian M, Jahani Hashemi H. Agents associated with candida vulvovaginitis in women referred to health centers in Qazvin. J Qazvin Uni Med Sci. 2007;11 (3):35-9.

8. Jamilian M, Mashadi E, Sarmadi F, Ghaznavi A, Bani Jamali M, Farhadi E, et al. Frequency of vulvovaginal candidiasis species in non-pregnant 15-50 years old women in spring 2005 in Arak. J Arak Uni Med Sci. 2007;2 (10):7-14

9. Khoursandi M, Modares GM, Khosravi A. Recovery and Recurrence of Vaginal Candidiasis after oral and Intravaginal Treatment. J Qazvin Uni Med Sci. 2000;14 (25):2.

10. Ramezan Zadeh F, Babaei GR, Fakhar F. A survey of oral contraceptive pills (LD) as a cause of Candidial vaginitis. Hakim Res J. 2001;1 (4):25-30.

11. Kamali F, Gharibi T, Naiemi B, Afshary P. Treatment of candida vaginitis: comparison of single and sequential dose of fluconazole. Iranian South Med J. 2003;6 (1):25-30.

12. Kariman NS, Shafaei Z, Afrakhteh M, Valaei N, Ahmadi M. Comparing fluconazole and clotrimazole in treatment of Candida albicans vaginiti. Behbood. 2002;14 (6):9-16.

13. Mohanty S, Xess I, Hasan F, Kapil A, Mittal S, Tolosa JE. Prevalence \& susceptibility to fluconazole of Candida species causing vulvovaginitis. Indian J Med Res. 2007;126 (3):216-9.

14. Momeni A, Shadzi S, Ghadeganipour M, Asilian A, Khorsandi R. The effect of itraconazole on treatment of vaginal candidiasis.J Kerman Uni Med Sci. 1995;2 (2):71-7.

15. Ramezan Zadeh F, Babaei GR, Karbalaei Mohammad S. Comparative study of efficacy of single-dose (500mg) and three days (200mg daily) treatment of candidial vulvovaginitis by vaginal tablets of Clotrimazole. Hakim Res J. 2000;2 (3):103-8.

16. Consolaro ME, Albertoni TA, Svidzinski AE, Peralta RM, Svidzinski TI. Vulvovaginal candidiasis is associated with the production of germ tubes by Candida albicans. Mycopathologia. 2005;159 (4):501-7.

17. Mahmoudabadi AZ, Najafyan M, Alidadi M. Clinical study of Candida vaginitis in Ahvaz, Iran and susceptibility of agents to topical antifungal. PakJ Med Sci. 2010;26 (3):607-10.

18. Pakshir K, Bahaedinie L, Rezaei Z, Sodaifi M, Zomorodian K. In vitro activity of six antifungal drugs against clinically important dermatophytes. Jundishapur J Microbiol. 2007;2 (4):158-63.

19. Pfaller MA, Diekema DJ, Colombo AL, Kibbler C, Ng KP, Gibbs DL, et al. Candida rugosa, an emerging fungal pathogen with resistance to azoles: geographic and temporal trends from the ARTEMIS DISK antifungal surveillance program. J Clin Microbiol. 2006;44 (10):3578-82.

20. Defontaine A, Bouchara JP, Declerk P, Planchenault C, Chabasse $\mathrm{D}$, Hallet JN. In-vitro resistance to azoles associated with mitochondrial DNA deficiency in Candida glabrata. J Med Microbiol. 1999;48 (7):663-70.

21. Al-Abeid HM, Abu-Elteen KH, Elkarmi AZ, Hamad MA. Isolation and characterization of Candida spp. in Jordanian cancer patients: prevalence, pathogenic determinants, and antifungal sensitivity. Jpn J Infect Dis. 2004;57 (6):279-84.

22. Quindos G, Abarca L, Carrillo-Munoz AJ, Arevalo MP, Bornay FJ, Casals JB, et al. Multicenter survey of in vitro antifungal resistance in yeasts of medical importance isolated from Spanish patients. Rev Iberoam Micol.1999;16 (2):97-100.

23. Pelletier R, Peter J, Antin C, Gonzalez C, Wood L, Walsh TJ. Emergence of resistance of Candida albicans to clotrimazole in human immunodeficiency virus-infected children: in vitro and clinical correlations. J Clin Microbiol. 2000;38 (4):1563-8.

24. Gianni C. Update on antifungal therapy with terbinafine. G Ital Dermatol Venereol. 2010;145 (3):415-24.

25. Barot BS, Parejiya PB, Patel HK, Gohel MC, Shelat PK. Microemulsion-based gel of terbinafine for the treatment of onychomycosis: optimization of formulation using D-optimal design. AAPS Pharm Sci Tech. 2012;13 (1):184-92.

26. Shivakumar V, Okade R, Rajkumar V, Sajitha K, Prasad SR. Intermittent pulse-dosed terbinafine in the treatment of tinea corporis and/or tinea cruris. Indian J Dermatol. 2011;56 (1):121-2. 